\title{
NUMERICAL ESTIMATES OF ELECTRODYNAMICS PROCESSES IN THE INDUCTOR SYSTEM WITH AN ATTRACTIVE SCREEN AND A FLAT RECTANGULAR SOLENOID
}

Purpose. To carry out numerical estimates of currents and forces in the investigated inductor system with an attractive screen (ISAS) and determine the effectiveness of the force attraction. Methodology. The calculated relationships and graphical constructions were obtained using the initial data of the system: induced current in the screen and sheet metal; the distributed force of attraction (Ampère force); the repulsive force acting on the sheet metal (Lorentzforce); amplitude values of the force of attraction and repulsion; phase dependence of the force of attraction, the repulsive force and the total resulting force. Results. The results of calculations in the form of graphical dependencies of electrodynamic processes in the region under the conductors of a rectangular solenoid of inductor system with an attracting screen are presented. The graphs of forces and currents in region of dent are obtained. In the paper the analysis of electrodynamics processes for whole area under the winding of inductor system with an attractive screen is shown. The flowing this processes in the region of dent a given geometry is presented. Originality. The considered inductor system with an attractive screen and a rectangular solenoid is improved, in comparison with the previous developed ISAS. It has a working area under the lines of parallel conductors in the cross section of a rectangular solenoid, and this allows to place a predetermined portion of the sheet metal anywhere within the working region. Comparison of the indicators of electrodynamics processes in the considered variants of calculation shows an approximate growth of almost 1.5 times the power indicators in the area of the accepted dent in comparison with similar values for the entire area under the winding of the ISAS. Practical value. The results obtained are important for the practice of real estimates of the excited forces of attraction. With a decrease in the dent, the amplitude of the electrodynamics forces being excited is growing proportionally (for example, when the depth decreases by $3 \mathrm{~mm}$, the forces increase more than 2 times). The highest efficiency of force attraction is for sheet metal regions, the dimensions do not exceed the transverse dimensions of the winding branches of the exciting solenoid. References 8 , figure 7.

Key words: magnetic-pulsed treatment, inductor system, attractive screen, rectangular solenoid, electrodynamics processes.

Проведены численные оценки и анализ полученных результатов для токов и сил в исследуемой индукторной системе с притягивающим экраном и прямоугольным соленоидом. Выявлено, что при уменьщении глубины вмятины в металле, амплитуда, возбуждаемых электродинамических усилий, растёт. Анализ показал, что значение сил отталкивания, интегральное во времени, ниже развиваемых сил притяжсения. При достаточно низких рабочих частотах действующих полей, рассмотренная система работает, как инструмент магнитно-импульсного притяжения тонкостенных листовых металлов. Библ. 8, рис. 7.

Ключевые слова: магнитно-импульсная обработка, индукторная система, притягивающий экран, прямоугольный соленоид, электродинамические процессы.

Introduction. The development of any area of modern industry is focused on the accelerated introduction of innovations. This applies to the metalworking area. The needs of society also indicate that it is necessary to promote a greater development of manufacturing industries. Magnetic-pulse processing of metals (MPPM) has recently become quite common in the application of various manufacturing operations [1, 2]. In this regard, the improvement of systems and tools that improve the efficiency of existing devices or the creation of absolutely new ones for the modern level of development of MPPM is actual.

Analysis of major achievements and publications. The main component of any system of magnetic-impulse attraction of thin-walled metal sheets is the inductor system. Its improvement is a solution to the problem of increasing the effectiveness of instruments of magnetic pulse technology. In order to obtain high homogeneity of the excited field and forces in the working area of the MPPM tool, inductor systems with an attractive screen (ISAS) were created. The principle of operation is based on the mutual attraction of conductors with identically directed currents (Ampere law) [3-6].

The goal of the work is to carry out numerical estimates of electrodynamics forces in the «inductor system with an attractive screen» under investigation to determine the effectiveness of the force attraction.
Problem definition. The ISAS system consists of a source of a magnetic field (a flat rectangular solenoid), an auxiliary conducting screen and an object of processing. The field of the solenoid excites unidirectional Foucault eddy currents in the screen and sheet metal. Due to the rigidly fixed screen, the attraction experiences only the processing object. Force magnetic-pulse action leads to a stretching of the specified area on its surface. The working zone of the ISAS under consideration is the area under the branches of the parallel conductors, that is, the specified section of the sheet metal processed object, which has a depression (for example, a dent), which must be deformed (attracted).

Initial data (Fig. 1). Solenoid:

a) number of turns in each branch $-N=10$;

b) branch width $-(b-a)=0.05 \mathrm{~mm}$;

c) width of turn and interturn insulation $g=\frac{(b-a)}{2 N-1} \approx 0,0026 \mathrm{~m}$;

d) internal solenoid window $-a=0.025 \mathrm{~m}$;

e) current in the inductor generated at the capacitive storage discharge $-C=1200 \mu \mathrm{F}$ with voltage $-U_{m} \approx 2000 \mathrm{~V}$ at frequency $-f=1500 \mathrm{~Hz}$, is assumed $-J_{m} \approx 22620 \mathrm{~A}$ (respectively, the linear density of the exciting current $\left.j_{m} \approx 8.6 \cdot 10^{6} \mathrm{~A} / \mathrm{m}\right)$.

(C) E.A. Chaplygin, M.V. Barbashova, A.Yu. Koval 


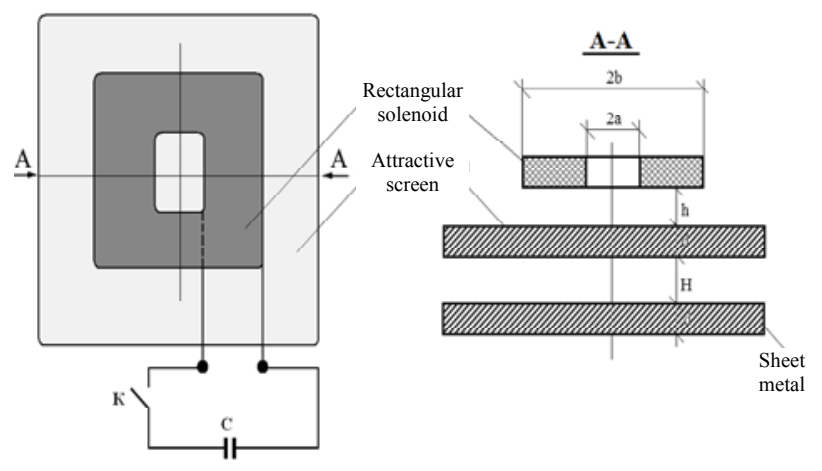

Fig. 1. Schematic representation of ISAS, $C$ - capacitance, $K-$ switch

Screen and sheet metal:

a) thickness $-d=0.0075 \mathrm{~m}$;

b) conductivity $-\gamma=0.4 \cdot 10^{7}(\Omega \cdot \mathrm{m})^{-1}$ (steel).

The system geometry:

a) distance between the working surface of the solenoid and the screen $-h=0.0005 \mathrm{~m}$;

b) dent depth $-H=0.005 \mathrm{~m}$, its length $-D=0.03 \mathrm{~m}$.

Calculation relations:

a) from the expression obtained in [7] we obtain the formula for the current induced in the screen:

$$
\begin{aligned}
& j_{\mathrm{e}}(\psi, y)=\left(\frac{J_{m}}{g}\right) \frac{\left(\mu_{0} \gamma \omega d^{2}\right)}{2} \frac{d j_{i}(\psi)}{d \psi} \times \\
& \times \int_{0}^{\infty} f(x) e^{-x \cdot \frac{h}{d}}\left(1-e^{-x}\right) \sin \left(x \frac{y}{d}\right) d x
\end{aligned}
$$

where $J_{m}$ is the amplitude of the current in the solenoid, $\psi=\omega t$ is the phase of the induced current in the pulse, $j_{i}(\psi)$ is the phase function of the current in the solenoid, $f(x)=\frac{2}{\pi} \cdot \frac{1}{x^{3}} \int_{0}^{\infty} f(\alpha) \cdot \sin (\alpha) d \alpha, \quad f(\alpha)$ is the lateral distribution function of the exciting current

$$
\begin{gathered}
f(\alpha)=\left\{\begin{array}{l}
1, \alpha \in\left[\frac{a}{d}, \frac{b}{d}\right], \\
0, \alpha \notin\left[\frac{a}{d}, \frac{b}{d}\right],
\end{array}\right. \\
f(x)=\frac{2}{\pi x^{3}} \cdot\left(\sum _ { k = 1 } ^ { N } \left[\cos \left(x \cdot\left(\frac{a}{d}+2(k-1) \frac{g}{d}\right)\right)-\right.\right. \\
\left.\left.-\cos \left(x \cdot\left(\frac{a}{d}+(2 k-1) \frac{g}{d}\right)\right)\right]\right) ;
\end{gathered}
$$

b) from the relation obtained in [7] we obtain a formula for the current induced in sheet metal:

$$
\begin{aligned}
& j_{\operatorname{lm}}(\psi, y)=\left(\frac{J_{m}}{g}\right) \frac{\left(\omega \mu_{0} \gamma d^{2}\right)}{2} \frac{d j_{i}(\psi)}{d \psi} \times \\
& \times \int_{0}^{\infty} f(x) e^{-x \frac{(h+d+H)}{d}}\left(1-e^{-x}\right) \sin \left(x \frac{y}{d}\right) d x
\end{aligned}
$$

c) from the relation obtained in [7] we find the distributed force of attraction (Ampere force),

$$
F_{\text {attr }}(\psi, y)=\frac{\mu_{0}}{2 \pi} \cdot j_{\mathrm{e}}(\psi, y) \cdot j_{\operatorname{lm}}(\psi, y) \cdot \frac{D}{H} ;
$$

where $D$ is the dimension (length), $H$ is the dent depth, $j_{\mathrm{e}, \mathrm{lm}}(\psi, y)$ is the current density induced in the screen and sheet metal in accordance with (1), (2);

d) the repulsive force (the Lorentz force!) acting on the sheet metal is determined from the expression in [7]:

$$
F_{\text {rep }}(\psi, y)=\frac{\mu_{0}}{2 \pi} \cdot j_{i}(\psi, y) \cdot j_{\operatorname{lm}}(\psi, y) \cdot \frac{D}{(h+d+H)},
$$

where $j_{i}(\psi, y)$ is the current density in the solenoid,

$$
j_{i}(\psi, y)=\left\{\begin{array}{cl}
\left(\frac{J_{m}}{g}\right) \cdot j_{i}(\psi), & \text { at }|y| \in[a, b], \\
0, & \text { at }|y| \notin[a, b],
\end{array}\right.
$$

$j_{i}(\psi)$ is the phase dependence of the current in the solenoid, $j_{\operatorname{lm}}(\psi, y)$ is the current density induced in sheet metal in accordance with (2);

e) amplitude-for-phase value of the attractive force

$$
\begin{aligned}
& F_{\mathrm{attr}}^{\max }(y)=\left.F_{\mathrm{attr}}(\psi, y)\right|_{\psi=\psi_{\max }}= \\
& =\left.\left.\frac{\mu_{0}}{2 \pi} \cdot j_{\mathrm{e}}(\psi, y)\right|_{\psi=\psi_{\max }} \cdot j_{\mathrm{lm}}(\psi, y)\right|_{\psi=\psi_{\max }} \cdot \frac{D}{H} ;
\end{aligned}
$$

f) phase dependence of the attractive force averaged in the transverse dimension on the interval $y \in[-b, b]$

$$
\bar{F}_{\text {attr }}(\psi)=\frac{1}{2 b} \int_{-b}^{b} F_{\text {attr }}(\psi, y) d y
$$

g) amplitude value of repulsive force

$$
\begin{aligned}
& F_{\text {rep }}^{\max }(y)=\left.F_{\text {rep }}(\psi, y)\right|_{\psi=\psi_{\text {max }}}= \\
& =\left.\frac{\mu_{0}}{2 \pi} j_{i}(\psi, y) j_{1 \mathrm{~m}}(\psi, y)\right|_{\psi=\psi_{\max }} \frac{D}{(h+d+H)}
\end{aligned}
$$

h) phase dependence of the repulsive force averaged in the transverse dimension on the interval $y \in[-b, b]$

$$
\bar{F}_{\text {rep }}(\psi)=\frac{1}{2 b} \int_{-b}^{b} F_{\text {rep }}(\psi, y) d y \text {; }
$$

i) phase dependence of the resultant force, found as a phase superposition of repulsive and attractive forces averaged in the transverse dimension along the width of the ISAS in the interval $y \in[-b, b]$

$$
\bar{F}_{\text {sum }}(\psi)=\bar{F}_{\text {attr }}(\psi)+\bar{F}_{\text {rep }}(\psi) .
$$

The characteristic phase dependence of the exciting aperiodic signal can be described by a function of the form [8]:

$$
j_{i}(\psi)=\left\{\begin{array}{l}
\sin \psi, \quad \psi \leq \frac{\pi}{2}, \\
\exp \left(-\beta \cdot\left(\psi-\frac{\pi}{2}\right)\right), \psi \geq \frac{\pi}{2}, \beta \approx 0,75 .
\end{array}\right.
$$


In addition, we illustrate graphical dependencies with additional averaged numerical estimates:

a) the value of the attractive force averaged in the transverse dimension on the interval $y \in[-b, b]$ and in phase $\psi \in[0,2 \pi]$

$$
\bar{F}_{\text {attr }}=\frac{1}{2 \pi} \int_{0}^{2 \pi} \bar{F}_{\mathrm{attr}}(\psi) d \psi=\frac{1}{(2 \pi \cdot 2 b)} \int_{0}^{2 \pi} \int_{-b}^{b} F_{\mathrm{attr}}(\psi, y) d y d \psi .
$$

b) the value of the repulsive force averaged in the transverse dimension on the interval $y \in[-b, b]$ and in phase $\psi \in[0,2 \pi]$

$$
\bar{F}_{\text {rep }}=\frac{1}{2 \pi} \int_{0}^{2 \pi} \bar{F}_{\text {rep }}(\psi) d \psi=\frac{1}{(2 \pi \cdot 2 b)} \int_{0}^{2 \pi} \int_{-b}^{b} F_{\text {rep }}(\psi, y) d y d \psi \text {. }
$$

c) the value of the resultant force (the superposition of the attractive force - Ampere and the repulsive force Lorentz) averaged in the transverse dimension on the interval $-y \in[-b, b]$ and in phase $-\psi \in[0,2 \pi]$

$$
\begin{aligned}
& \bar{F}_{\text {sum }}=\frac{1}{2 \pi} \int_{0}^{2 \pi} \bar{F}_{\text {sum }}(\psi) d \psi=\frac{1}{(2 \pi \cdot 2 b)} \times \\
& \times \int_{0}^{2 \pi} \int_{-b}^{b} F_{\text {sum }}(\psi, y) d y d \psi .
\end{aligned}
$$

We note that formula (13) illustrates the quantitative increase in the efficiency of the magnetic-pulse attraction taking into account the phase of the proceeding process. Numerical estimates can be averaged over any interesting spatial or phase interval. For example, when considering processes in the region directly under a separate inductor current lead, the averaging interval $-y \cup[a,(a+b)]$.

\section{Results of calculations.}

1. Electrodynamics processes in the region under the conductors of a rectangular solenoid of ISAS (Fig. 2-7).

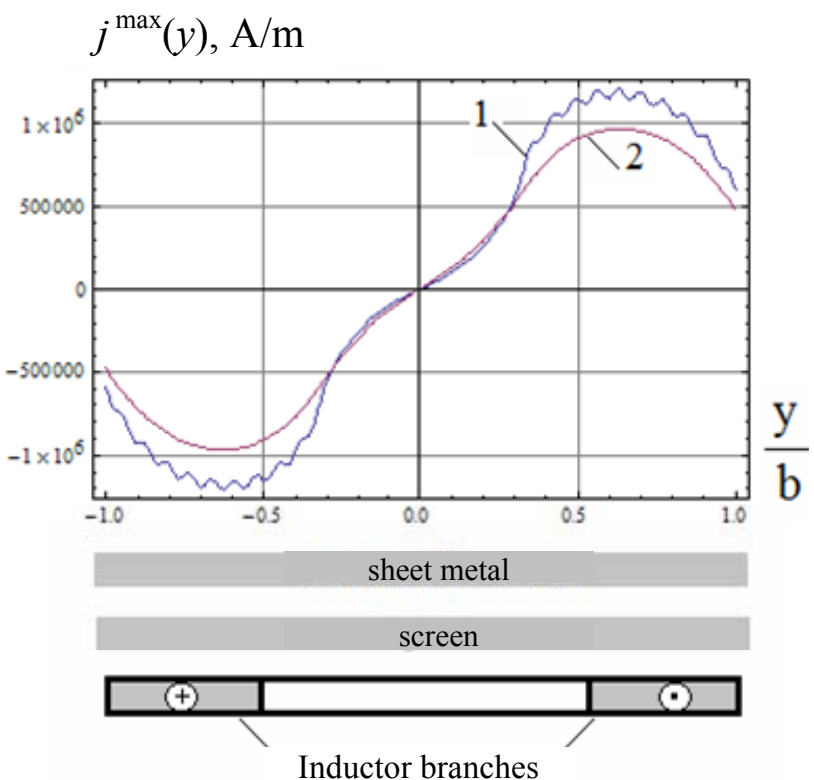

Fig. 2. The picture of the spatial distribution of induced currents, 1 - screen metal, 2 - sheet metal

With a decrease in the depth of the dent, the amplitude of the excited electrodynamics forces increases in practice, in proportion. Thus, with a decrease in depth from $0.005 \mathrm{~m}$ to $0.002 \mathrm{~m}$, the forces increase by more than a factor of $\sim 2$.

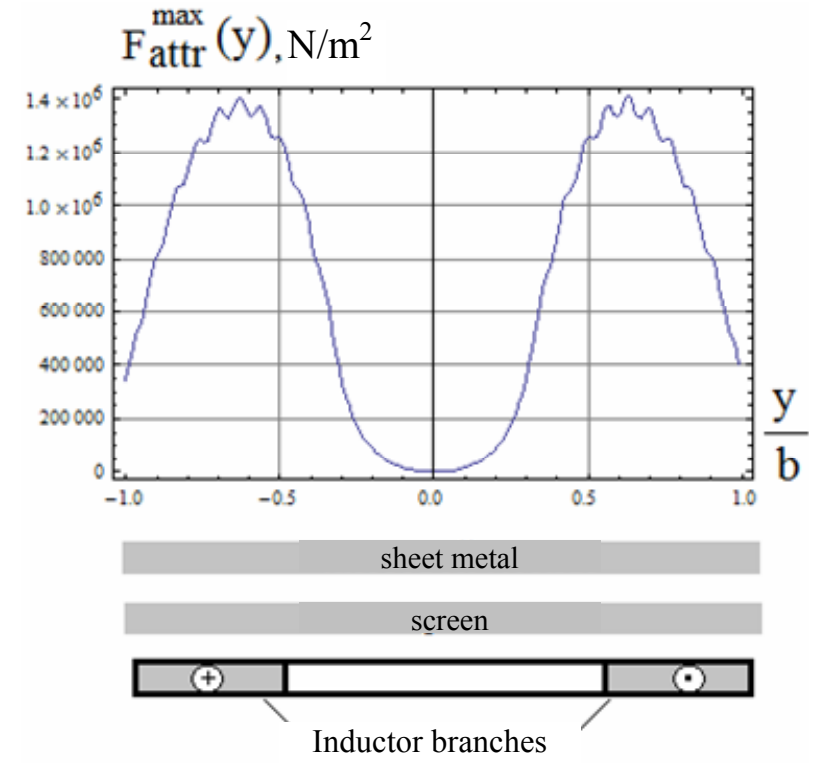

Fig. 3. The spatial pattern of the distributed force of attraction (Ampere), the average value on the width of the ISAS -

$$
\bar{F}_{\text {attr }} \approx 0.78 \cdot 10^{6} \mathrm{~N} / \mathrm{m}^{2}
$$

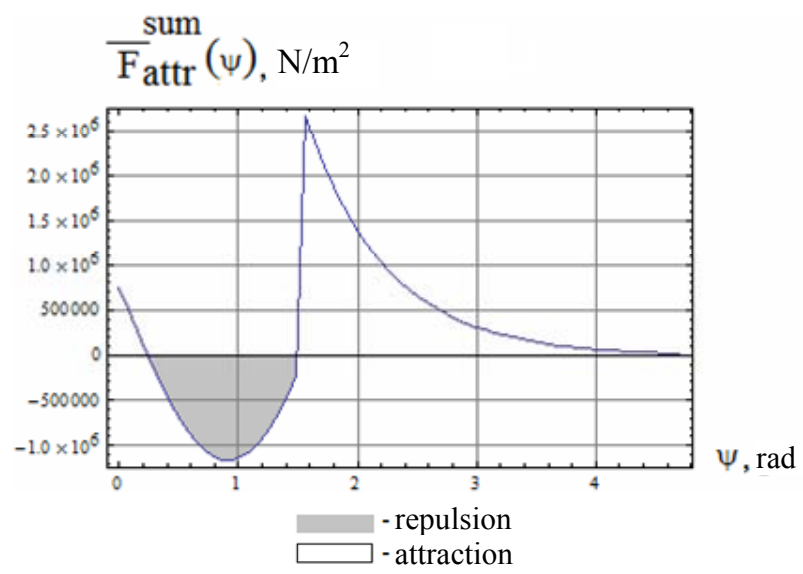

Fig. 4. The phase dependence of the resultant force (superposition of Ampere and Lorentz) acting on the sheet metal in the ISAS, the average value over the time of action -

$$
\bar{F} \operatorname{sum}_{\text {attr }} \approx 0.245 \cdot 10^{6} \mathrm{~N} / \mathrm{m}^{2}
$$

The main results of the calculations are as follows:

- calculated data are integral indicators of electrodynamics processes, characterizing their flow over the entire area of the inductor system, excited by a multiturn flat rectangular solenoid with current amplitude in the winding of $\sim 22.6 \mathrm{kA}$ with storage capacitor voltage of $\sim 2 \mathrm{kV}$ and storage energy of $\sim 2.4 \mathrm{~kJ}$;

- the temporal dependencies of the exciting and induced currents are different, which ultimately causes the oscillation of the Lorentz force, that is, the appearance of repulsion and attraction intervals;

- the prevalence of repulsive forces over the forces of attraction is short-term and occurs exclusively in the initial phase of the process of force action;

- averaging of the excited forces over the action time shows more than an order of magnitude ( 15 times $)$ the 
excess of attraction over repulsion (attraction $-\bar{F}_{\text {attr }} \approx$ $\approx 0.282 \cdot 10^{6} \mathrm{~N} / \mathrm{m}^{2}$, repulsion $-\bar{F}_{\text {rep }} \approx-0.0182 \cdot 10^{6} \mathrm{~N} / \mathrm{m}^{2}$ );

- in the integral (averaging over the action time and the spatial variable), the ISAS examined over its entire area provides a magnetic-pulse attraction with force $\bar{F}$ sum $_{\text {attr }} \approx 0.245 \cdot 10^{6} \mathrm{~N} / \mathrm{m}^{2}$.

2. Currents and forces in the area of the dent.

Geometry of the dent area (assumed area of force action): $D \times D \times H=0.03 \mathrm{~m} \times 0.03 \mathrm{~m} \times 0.05 \mathrm{~m}$.

The specified sheet metal area is placed under the plane of the conductors of any of the branches of a rectangular solenoid, which excites the «inductor system with an attractive screen» (ISAS).

For the assumed region of force action, averaging over the transverse spatial variable is performed in the interval $\psi \in[0, D]$.

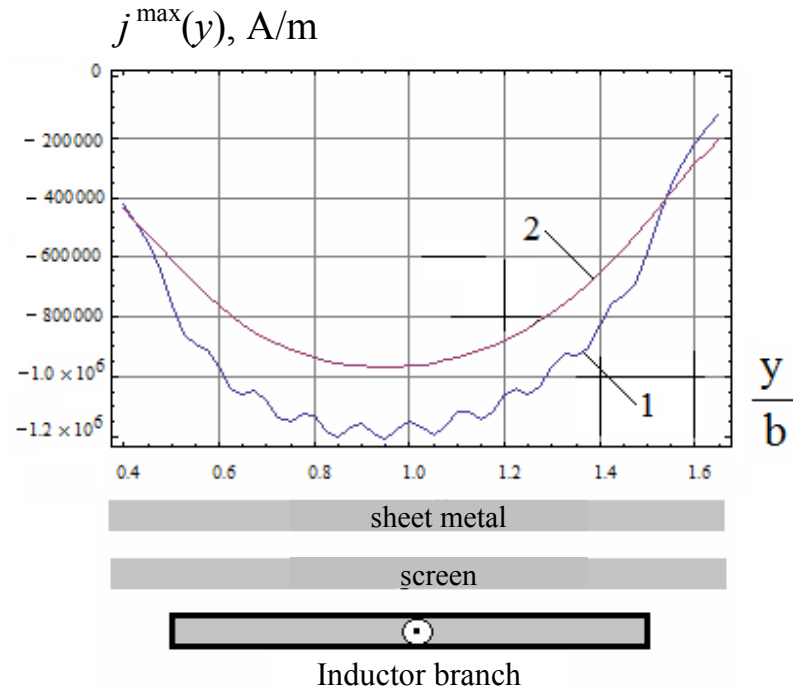

Fig. 5. The picture of the spatial distribution of induced currents, 1 - screen metal, 2 - sheet metal

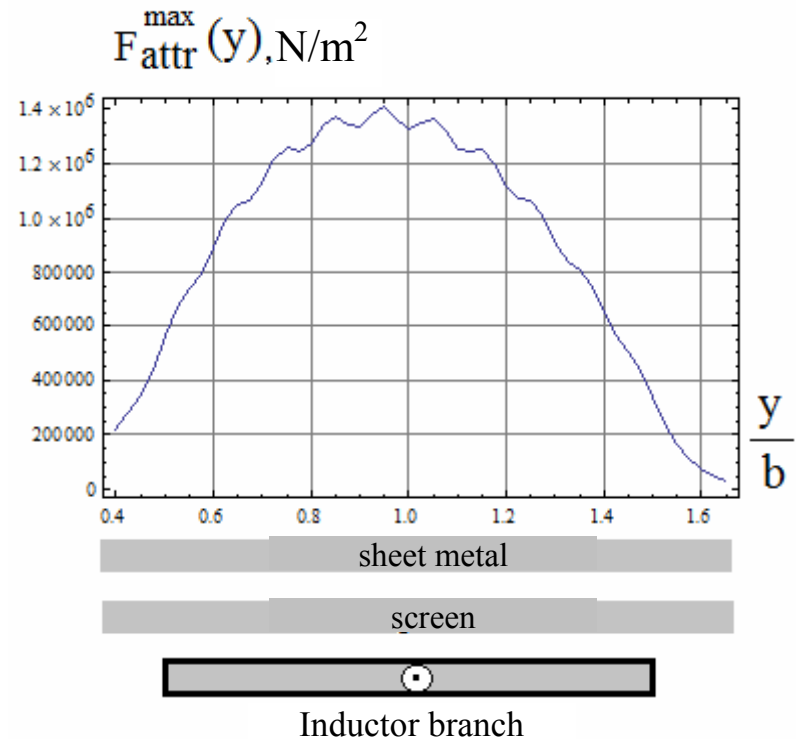

Fig. 6. The spatial pattern of the distributed force of attraction (Ampere), the average value on the width of the ISAS -

$$
\bar{F}_{\text {attr }} \approx 0.92 \cdot 10^{6} \mathrm{~N} / \mathrm{m}^{2}
$$

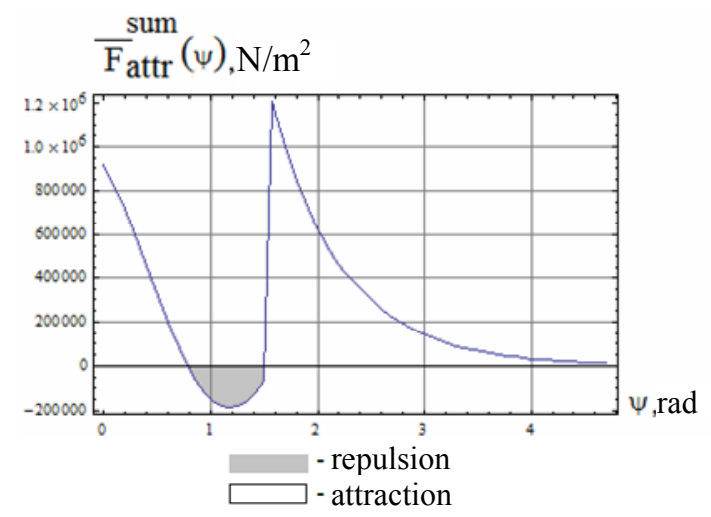

Fig. 7. The phase dependence of the resultant force (superposition of Ampere and Lorentz!) acting on the sheet metal in the ISAS, the average value over the time of action

$$
-\bar{F} \text { attr } \approx 0.345 \cdot 10^{6} \mathrm{~N} / \mathrm{m}^{2}
$$

Discussion of the results of calculations:

- calculated data are local indicators of electrodynamics processes that characterize their flow in the region of a dent of a given geometry, in contrast to previous results obtained for the entire area under the ISAS winding;

- as before, the prevalence of repulsive forces over the forces of attraction is short-term and occurs exclusively in the initial phase of the process of force action;

- averaging of the excited forces by time of action shows a significant excess of attraction over repulsion (attraction - $\bar{F}_{\text {attr }} \approx 0.35 \cdot 10^{6} \quad \mathrm{~N} / \mathrm{m}^{2}$, repulsion $\bar{F}_{\text {rep }} \approx-0.056 \cdot 10^{6} \mathrm{~N} / \mathrm{m}^{2}$ );

- in the integral (averaging over the action time and the spatial variable), the ISAS considered on its entire area provides a magnetic-pulse attraction with a distributed force $-\bar{F}_{\text {attr }}^{\text {sum }} \approx 0.345 \cdot 10^{6} \mathrm{~N} / \mathrm{m}^{2}$;

- in general, the comparison of the indicators of electrodynamics processes in the considered variants of the calculation shows an approximate growth in 1.3-1.5 times of the force indicators in the area of the accepted dent on the surface of the sheet metal in comparison with similar values for the whole area under the winding of the ISAS inductor.

\section{Conclusions.}

1. A numerical analysis of electrodynamics processes in an inductor system with an attractive screen and a source of a magnetic field - a flat rectangular solenoid is carried out in the work.

2. Numerical estimates have shown that when the depth of the dent decreases, the amplitude of the electrodynamics forces excited increases in practice in proportion.

3. The greatest efficiency of force attraction occurs for sheet metal regions, the dimensions of which do not exceed the transverse dimensions of the winding branches of the exciting solenoid.

4. The integral value of the repulsive forces in time is much lower than the developed attractive forces (less than $\sim 5 \%$ ).

5. In general, at sufficiently low operating frequencies of acting fields $(\sim 1500 \mathrm{~Hz})$, the «inductor system with an 
attractive screen» considered operates as an instrument of magnetic-pulse attraction of thin-walled sheet metals. Local distributed forces of attraction in the region of given areas (for example, dents with limited dimensions!) at current in the inductor of $\sim 22 \mathrm{kA}$ can reach values of the order of $\sim 0.345 \cdot 10^{6} \mathrm{~N} / \mathrm{m}^{2}$.

\section{REFERENCES}

1. Batygin Yu.V., Lavinskiy V.I., Khimenko L.T. Impul'snyye magnitnyye polya dlya progressivnykh tekhnologiy. Tom 1. Izdaniye vtoroye, pererabotannoye $i$ dopolnennoye. [Pulsed magnetic fields for advanced technologies. Vol.1. 2nd edition, revised and enlarged.] Kharkov, MOST-Tornado Publ., 2003. 284 p. (Rus).

2. Yuriy V. Batygin, Sergey F. Golovashchenko, Andrey V. Gnatov. Pulsed electromagnetic attraction of sheet metals fundamentals and perspective applications. Journal of Materials Processing Technology, 2013, vol.213, no.3, pp. 444-452. doi: 10.1016/j.jmatprotec.2012.10.003.

3. Psyk V., Risch D., Kinsey B.L., Tekkaya A.E., Kleiner M. Electromagnetic forming - A review. Journal of Materials Processing Technology, 2011, vol.211, no.5, pp.787-829. doi: 10.1016/j.jmatprotec.2010.12.012.

4. Gnatov A., Argun S. New method of car body panel external straightening. Tools of method. International Journal of Vehicular Technology, 2015, vol.2015, pp. 1-7. doi:

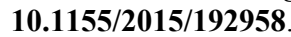

5. Batygin Yuri V., Sergey F. Golovashchenko, Andrey V. Gnatov. Pulsed electromagnetic attraction of nonmagnetic sheet metals. Journal of Materials Processing Technology, 2014, vol.214, iss.2, pp. 390-401. doi: 10.1016/j.jmatprotec.2013.09.018.

6. Batygin Yu.V., Golovashchenko S.F., Gnatov A.V., Chaplygin E.A. Pulsed Electromagnetic Attraction Processes for Sheet Metal Components. Proceedings of the $6^{\text {th }}$ International Conference High Speed Forming 2014, May 26-29, 2014, Daejeon, Korea, pp. 253-260.

7. Batygin Yu.V., Chaplygin E.A., Shinderuk S.A., Gavrilova O.E. Inductor system with attracting screen and rectangular solenoid. Automobile Transport, 2017, no.41, pp. 146-154. (Rus).

8. Chitode J.S. Signal an systems. Pune, India, Technical Publications, 2009. 767 p.

Received 25.12.2017

\section{E.A. Chaplygin ${ }^{1}$, Candidate of Technical Sciences, Associate} Professor,

M.V. Barbashova ${ }^{1}$, Candidate of Technical Sciences,

A.Yu. Koval ${ }^{1}$, student,

${ }^{1}$ Kharkiv National Automobile and Highway University, 25, Yaroslava Mudrogo Str., Kharkov, 61002, Ukraine, phone +380 577073727 ,

e-mail: chaplygin.e.a@gmail.com; barbashova1987@gmail.com

How to cite this article:

Chaplygin E.A., Barbashova M.V., Koval A.Yu. Numerical estimates of electrodynamics processes in the inductor system with an attractive screen and a flat rectangular solenoid. Electrical engineering \& electromechanics, 2018, no.2, pp. 54-58. doi: 10.20998/2074-272X.2018.2.09. 